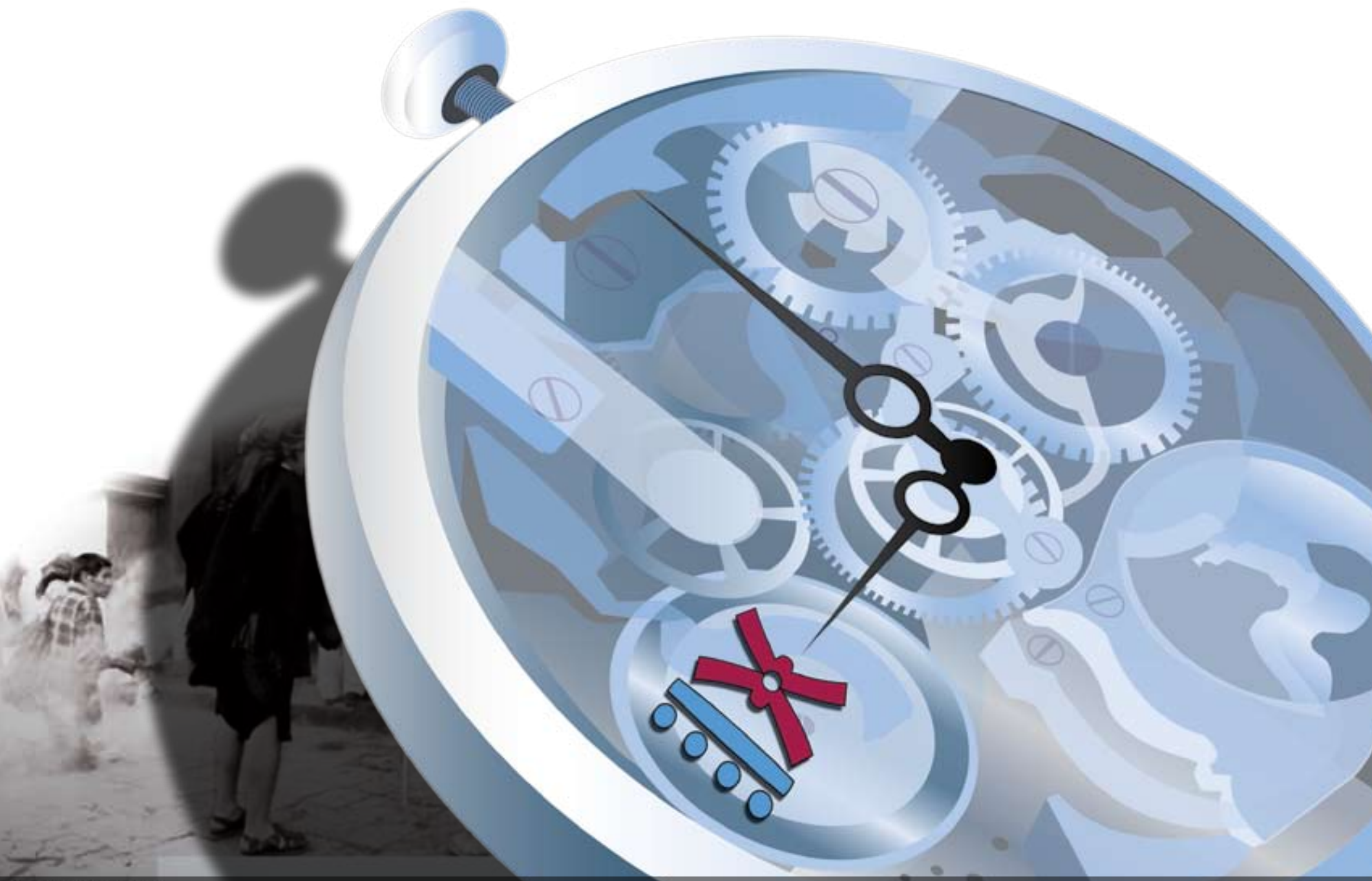

\section{$9^{\circ}$ C O N G R E S O 2. CENTROAMERICANO DE H IS TOR I A}

ISSN 1409-469X

Fecha de recepción: 15 de mayo 2008 Fecha de aceptación: 30 de mayo 2008

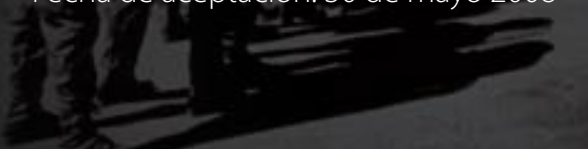

Historia, paradigmas y modelos de atención de salud: reflexiones desde la perspectiva brasileña

Miembros del Consejo Editorial:

Dr. Ronny Viales, Dr. Juan José Marín

Editores Técnicos:

Allan Fonseca, Andrés Cruz, Gabriela Soto

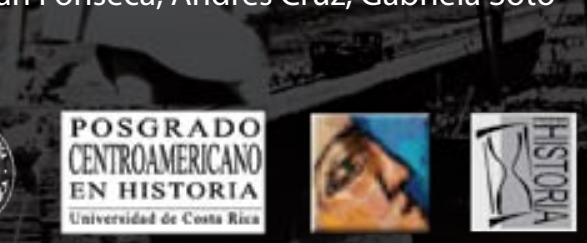


Indexaciones: Repositorio de Revistas UCR, DIALNET, Latindex, REDALYC Directorio y recolector de recursos digitales del Ministerio de Cultura de España, Directory of Open Access Journals. Diálogos Revista Electrónica de Historia ISSN 1409-469X. Número especial 2008. Dirección web: http://historia.fcs.ucr.ac.cr/dialogos.htm

\title{
Historia, paradigmas y modelos de atención de salud: reflexiones desde la perspectiva brasileña
}

\author{
Aldira Guimarães Duarte Domínguez \\ Doutora em ciências da saúde pela Universidade de \\ Brasília, Mestre em estudos sociais e políticos Latino- \\ americanos. Graduação em fisioterapia pela universidade \\ estadual da Paraíba. Especialista em acupuntura pelo \\ Instituto Mineiro de Estudos Sistêmicos. Atualmente \\ é coordenadora do curso de fisioterapia do Centro \\ Universitário Unieuro e professora visitante da \\ Universidade de Brasília. E-mail: aldira_guimaraes@ \\ yahoo.com.br
}


Introdução

Existem hoje basicamente dois modelos ou paradigmas de assistência à saúde. O paradigma biomédico e o da promoção da saúde. No paradigma biomédico as doenças são estudadas a partir dos mecanismos biológicos. Os estudiosos dedicam-se somente a definir a doença, entender o processo pelo qual ela age, identificando sua causa especifica, para a partir de então, buscarem os procedimentos de tratamento mais indicado. É o modelo hegemônico.

O paradigma de promoção da saúde consiste no processo de capacitação da comunidade para atuar na melhoria da sua qualidade de vida e saúde, incluindo uma maior participação no controle deste processo Consagra como pré-requisitos para saúde: a paz, educação, habitação, poder aquisitivo, ecossistema estável, conservação dos recursos naturais, justiça social e equidade. Nesse estudo, se fará um levantamento dos principais aspectos favoráveis e desfavoráveis dos dois modelos existentes. Espera-se ao final esclarecer o leitor sobre aspectos relevantes referente à temática.

\section{Conceito de Paradigma}

Existe um consenso generalizado entre acadêmicos de muitas, por não dizer todas, disciplinas científicas sobre o significativo aporte que Thomas Kuhn realizou à história e filosofia das ciências durante o século XX, particularmente no que se refere a introdução da noção de paradigma.

Inicialmente, Kuhn entendeu os paradigmas como realizações científicas universalmente reconhecidas que, durante certo tempo, proporcionam modelos de problemas e soluções a uma comunidade científica. Dessa maneira, a noção de paradigma incluiria um amplo espectro de fatores que incidiriam no fazer científico, incluindo leis, teorias, modelos, metodologias, crenças, valores, relações de poder, etc. Posteriormente, na Pós-data de 1969, Kuhn reformulou sua noção de paradigma em dois sentidos:

Por uma parte significa toda a constelação de crenças, valores, técnicas, etc., que compartem os membros de uma comunidade dada. Por outra parte, denota uma espécie de elemento de tal constelação, as concretas soluções de problemas que empregadas como modelos ou exemplos, podem substituir regras explícitas como 
base de solução dos restantes problemas da ciência normal ${ }^{1}$.

A reformulação da noção de paradigma kuhniano supões colocar a ênfase da sua proposta em duas idéias relativamente novas: matriz disciplinaria e modelos exemplares. Antes de analisar ambas idéias parecer fundamental se perguntar sobre qual a utilidade operacional dos paradigmas. Para Masterman², os paradigmas são particularmente úteis para uma comunidade científica quando não existem teorias ou generalizações formais consolidadas ao interior de alguma disciplina como é o caso da saúde coletiva e outras disciplinas da ciências da saúde. Neste caso, os paradigmas funcionam como um conjunto de hábitos que permitem sustentar o esforço rigoroso e reflexivo das diferentes disciplinas científicas. Eckberg e Hill ${ }^{3}$, concordam com Masterman ao concluírem que os paradigmas são corpos unificados de crenças compartidos por uma coesiva comunidade científica.

$\mathrm{Nef}^{4}$, destaca a capacidade hegemônica dos paradigmas, ele enfatiza as condições e possibilidades de convocatória e convencimento de cada articulação discursiva relativamente coerente, por parte de uma comunidade científica e tecnológica como por exemplo: acadêmicos, profissionais, para-profissionais, extensionistas de discursos.

Parece racionável compreender que a função central dos paradigmas é constituir-se em ferramentas de análise para orientar e dar coerência aos problemas de investigação das diferentes disciplinas científicas incluído-se aí a saúde coletiva. Aceitar a noção de paradigma desta maneira supõe importantes conseqüências que serão analisadas mais adiante.

Um dos aportes mais significativos e controvertidos da proposta kuhniana é a sua interpretação da estrutura comunitária da ciência. Para Kuhn, uma comunidade científica consiste nas pessoas que praticam uma especialidade científica, isto supõe um processo de educação e iniciação científica similar, absorção de bibliografia especializada, relativa competência,

1 Kuhn, T. La Estructura de las Revoluciones Científicas. Cidade do México: Fondo de Cultura Económica, 1999.

2 Masterman, M. The nature of pardigm. In: Katatos e Musgrave: Criticism and the Growth of knowfedge. Cambridge: Cambridge University Press. 1981 : pp 59-89

3 Eckberg e Hill. The paradigm concept and sociology: A critical Review . In Gary Gutiting.: Pardigmse revolutions. University of Notre Dame, 1980: pp. 117-136

4 Nef, J. "Paradigmas Emergentes em Políticas Sociales: Una Perspectiva Para el Análises Comparativo de las Políticas Públicas”. Revista Chilena de Administración Pública, № 8, Santiago do Chile: Colegio de Administradores Públicos de Chile, 1995, pp.5-25. 
compromisso profissional, coerência nos pontos de vista, etc. Em conseqüência, para Kuhn ${ }^{5}$ :

Os membros de uma comunidade científica se vêem a si mesmo, e são considerados por outros como os homens [e mulheres] exclusivamente responsáveis da investigação de todo um conjunto de objetivos comuns, que inclui a preparação de seus próprios sucessores. Dentro de tais grupos, a comunicação é quase plena, e o juízo profissional é relativamente unânime.

Obviamente, existem diferentes comunidades científicas, não obstante, todas e cada uma delas devem pertencer a algum paradigma. A comunidade científica mais ampla incluiria a todos os investigadores do planeta; num segundo nível se classificariam os científicos segundo a natureza de sua disciplina por exemplo: pesquisadores das ciências da saúde em geral, logo em comunidade de sub-disciplinas como: pesquisadores na área da fisioterapia e assim sucessivamente até chegar a micro-comunidades estruturadas de ciência, muitas vezes compostas por relativamente poucos, mais muito bem especializados, membros que normalmente atuam nas fronteiras do conhecimento como os investigadores de políticas de saúde.

Sobre a base destas ponderações, cabe fazer uma relação dos paradigmas que, no contexto de uma matriz disciplinaria é entendido como campo de tensão epistémica entre paradigmas ativos e em competências, permitem aproxima-se aos problemas de investigação da saúde coletiva. Aceitando o risco de parecer reducionista ou demasiado esquemático, é possível identificar ao menos duas posições teórico-metodológicas básicas ou paradigmas que formam parte da matriz disciplinaria para o estudo das temáticas relacionada com a saúde coletiva, incluindo a problemática da fisioterapia coletiva que é o que mais interessa para este estudo. ${ }^{6}$ Ao mesmo tempo, parece importante advertir que a presente proposta de matriz disciplinaria para a saúde coletiva ou mais especificamente para a fisioterapia coletiva, de modo algum pode ser considerada fechada ou completa. Na realidade reconhecendo seu caráter dinâmico ou dialético, esta matriz disciplinaria se apresenta aberta e flexível tanto a outros paradigmas que não tem sido considerado neste estudo como bem a críticas de outros especialistas na matéria.

$5 \quad$ Kuhn, T. La Estructura de las Revoluciones Cientificas. Cidade do México: Fondo de Cultura Económica, 1999.

6 Parece importante constatar que uma das pré-condições na construção da matriz disciplinaria para a saúde coletiva é reconhecer seu caráter "pré-paradigmatico" (no sentido kuhniano do termo). Isso supõe, entre outras consequências, ter consciência de suas condições limitações e possibilidades (inclusive no que se refere especificamente a sua matriz disciplinaria). 
Assim, não parece incorreto propor uma matriz disciplinaria para a saúde coletiva, entendendo matriz disciplinaria como campo de tensão epistémica entre paradigmas coexistentes, em competência, e, que ademais permite aproximar-se cientificamente aos problemas de investigação da especialidade composta, entre outros, por duas posturas teóricos- metodológicas ou paradigmas: o paradigma biomédico e o paradigma da promoção da saúde.

\section{O Paradigma Biomédico}

Surgiu no século XVII, com o francês Descartes que criticava arduamente a concepção de tratamento das doenças fundamentada na interação de ações que envolviam corpo, mente e espírito. Descartes, em sua filosofia, pregava a necessidade de uma maior aproximação das pesquisas da área médica com o corpo, visto por ele como uma máquina e que portanto, precisava de concertos quando apresentasse algum defeito ${ }^{7}$.

No paradigma biomédico, as doenças são estudadas somente a partir dos mecanismos biológicos, não sendo levando em consideração aspectos externos como por exemplo: maus hábitos de vida, estado emocional, meio ambiente, estado nutricional, dentre outros. Em outras palavras, neste modelo, os estudiosos e pesquisadores dedicam-se somente a definir a doença, entender o processo pelo qual ela age, identificando sua causa especifica, pois acreditam na unicausalidade da doença, para a partir de então atuarem sobre ela, buscando o procedimento de tratamento mais indicado.

Sobre o paradigma biomédico $\mathrm{Capra}^{8}$ faz a seguinte observação:

A ciência médica limitou-se à tentativa de compreender os mecanismos biológicos envolvidos numa lesão em algumas das várias partes do corpo. Esses mecanismos são estudados do ponto de vista da biologia celular e molecular, deixando de fora todas as influências de circunstâncias não biológicas sobre os problemas biológicos. Em meio à enorme rede de fenômenos que influenciam a saúde, a abordagem biomédica estuda apenas alguns aspectos fisiológicos. O conhecimento desses aspectos é, evidentemente, muito útil, mas eles representam apenas uma pequena parte da história. A pratica médica, baseada em tão limitada abordagem, não é muito eficaz na promoção e manutenção da boa saúde.

Entre os precursores do paradigma biomédico ademais de Descartes, se pode destacar: William Harvey (século XVII), pesquisador que utilizou o paradigma mecanicista para explicar o processo como ocorria a circulação sanguínea no corpo humano; Claude Bernard (século

7 Capra, F. O ponto de mutação/ A Ciência, a Sociedade e a Cultura emergente. São Paulo: Cutrix, 2001.

8 Capra, F. O ponto de mutação/ A Ciência, a Sociedade e a Cultura emergente. São Paulo: Cutrix, 2001 
XIX), estudioso dos processos fisiológicos do corpo humano; Rudolf Virchow (século XIX), pesquisador que colocou a biologia celular como a base da ciência médica ao perceber a presença de alterações celulares após a manifestação de quadros patológicos; e, Louis Pasteur (XIX), autor da teoria microbiana que prova a associação existente entre algumas doenças e micróbios específicos.

Como se pode constatar, a contribuição deste paradigma para o avanço da medicina é inquestionável, a evolução das pesquisas biológicas baseada na concepção mecanicista de Descartes avançou gradativamente passando do estudo de órgãos e corpo, para a célula e finalmente para as moléculas, criando ao menos três aspectos que não podem passar despercebidos: a) a tendência cada vez mais acentuada à especialização dos pesquisadores em partes cada vez menores do corpo, perdendo-se a visão do todo, b) a exigência do uso de alta tecnologia para o desenvolvimento das pesquisas tornando os custos da medicina muitas vezes insustentável e c) a responsabilidade sobre a saúde do paciente é transferida para o médico, ou seja, acreditase única e exclusivamente em sua capacidade para resolver qualquer problema de saúde que eventualmente venha a acometer o indivíduo, independente das condições psicossociais e cultural que este esteja inserido.

Entre as principais virtudes deste paradigma se destacam:

- Possibilitou o conhecimento minucioso e detalhado de quase todo corpo humano e sua fisiologia;

- Estudou e identificou varias patologias, entre elas as de caráter infecciosas, produzindo os medicamentos e as vacinas usadas em seu combate, acentuando seu declínio e em alguns casos até a erradicação;

- Favoreceu o estudo dos mecanismos celulares e moleculares responsáveis em grande parte pelo êxito e ascensão da medicina científica durante o século XX;

- Propiciou grandes descobertas na área da farmacologia como: a penicilina, insulina, pílula anticoncepcional, psicoativos, tranquilizantes e antidepressivos, entre outros, que revolucionaram o tratamento de várias doenças;

- Promoveu o avanço tecnológico na área médica que culminou com a possibilidade real da realização de transplantes de órgãos humanos. 
Como fragilidades desse paradigma destacam-se as seguintes:

- Analisa o corpo humano a partir da concepção corpo maquina, dedicando seus estudos em partes cada vez mais específicas do corpo, preocupando-se somente com o processo de cura, sem considerar a influencia de fatores externos nas condições de saúde das pessoas.

- Por se dedicar a estudar e entender partes cada vez menores do corpo humano, muitas vezes desconhecem os efeitos que os fármacos usado no tratamento destas partes especificas podem vir a provocar em outras regiões do corpo - são os chamados efeitos colaterais.

- Resulta em um modelo médico de alto custo, devido principalmente a dependência da medicina em relação à tecnologia. Sem esquecer que o uso da tecnologia por si só, não tem conseguido resolver os problemas de imperícia, negligencia e inacessibilidade aos centros assistenciais.

- Entende a saúde somente como a ausência de doença, deste modo, os serviços primários de atenção à saúde, assemelham-se mais a uma ilha auto-suficiente no seio da sociedade, do que a porta de entrada dos sistemas de saúde.

- Consiste em um paradigma fragmentado, caracterizado principalmente pelo predomínio de programas impostos de cima para baixo sem a participação da sociedade, sendo toda responsabilidade do setor saúde transferida para a categoria médica.

$\mathrm{Na}$ atualidade, o debate acadêmico e científico sobre o modelo médico de atenção a saúde está girando em torno a aspectos bem mais amplos do que o proposto pelo paradigma biomédico. Nesse novo enfoque, a saúde passa a ser entendida não só como a mera ausência de doença, envolvendo preocupações que abrangem desde os cuidados individuais com a saúde, às condições socio-econômicas em que vivem as pessoas. Isso devido principalmente à constatação da prevalência de um quadro de morbidade e mortalidade marcada pela combinação das doenças do subdesenvolvimento com as doenças do desenvolvimento.

Em sínteses parece evidente que o paradigma biomédico que tem sido muito importante a partir do século XVII, continua sendo sumamente influente e significativo para importantes setores da comunidade estruturada da ciência da saúde. Uma vez considerada suas principais virtudes, assim como, algumas debilidades, certamente o peso específico do paradigma biológico não pode ser ignorado ao interior do que se tem denominado de matriz disciplinaria da saúde coletiva, entendida como: campo de tensão epistémica entre paradigmas ativos e em competências. 


\section{O Paradigma da Promoção da Saúde}

Foi mencionado pela primeira vez em 1945 pelo historiador médico Henry Sigerist que considerava que: “[...] a saúde se promove quando se facilita um nível de vida decente, boas condições de trabalho, educação, cultura física, descanso e recreação”. ${ }^{9} \mathrm{O}$ pensamento deste médico historiador se propagou ao longo do tempo alcançado grandes proporções entre os pesquisadores e cientistas comprometidos com a temática da saúde coletiva chegando a se consolidar na atualidade como um dos paradigma predominantes no que se refere à atenção e cuidados em saúde.

Convém agregar que hoje, o paradigma da promoção da saúde, entendida como: o processo de capacitação da comunidade para atuar na melhoria da sua qualidade de vida e saúde, incluindo uma maior participação no controle deste processo ${ }^{10}$. Constitui uma das principais diretrizes impulsionada pela Organização Mundial da Saúde, para se alcançar maior desenvolvimento social, econômico e pessoal.

Historicamente, o acontecimento que mais marcou essa nova tendência na forma de se pensar a saúde foi a elaboração do Relatório Lalonde pelo epidemiologista canadense Marc Lalonde quando exercia o cargo de Ministro da Saúde deste país em $1974^{11}$. Em dito relatório, publicado pelo governo canadense em 1974, esse médico, coloca a promoção da saúde como uma das estratégias básica para a melhoria das condições de vida da população e da formação de capital social. O documento faz criticas ao fato de se utilizar somente às estatísticas de mortalidade para se determinar às prioridades de saúde do seu país. Ademais, defende que a queda nas taxas de mortalidade infantil e o aumento na expectativa de vida da população não deveria ser atribuída somente ao processo de universalização da assistência médica adotada no país, isso devido, alguns estudos confirmarem que estes indicadores modificavam-se dependendo da classe social que o indivíduo estava inserido ${ }^{12}$.

Esta nova forma e concepção de articulação da saúde com as condições sociais, econômicas e culturais em que os indivíduos estão inseridos no seu cotidiano, serviu de marco e referência para a Primeira Conferência Internacional Sobre Promoção da Saúde, realizada no Canadá, na cidade de Ottawa, em 1986. Entre os principais precursores do paradigma da promoção da saúde destacam-se: John A. Ryle, primeiro professor de medicina social da Universidade de Oxford; Henry Sigerist, que colocou a promoção da saúde entre as quatro tarefas da medicina; Marc Lalonde, autor do relatório Lalonde e em um contexto mais amplo, a Primeira Conferência 9 Ferraz, S. T. cidades saudáveis: uma urbanidade pra 2000. Brasília: Editora paralelo 15: 1999.

10 Ministério da saúde. Assistência hospitalar no SUS - 1995/1999, Brasília: Governo Federal/Ministério da Saúde, 2000.

11 Ferraz, S. T. cidades saudáveis: uma urbanidade pra 2000. Brasília: Editora paralelo 15: 1999.

12 Ibid. 
Internacional Sobre Promoção da Saúde em Ottawa, que culminou com a Carta de Ottawa que: consagra como pré-requisitos para a saúde: a paz, a educação, a habitação, o poder aquisitivo, um ecossistema estável a conservação dos recursos naturais, a justiça social e a equidade ${ }^{13}$.

Como se pode observar, no paradigma da promoção da saúde a preocupação dos pesquisadores e estudiosos não se concentrava somente nas intervenções médicas curativas de alta complexidade. Aí prevaleceu uma visão mais holística da saúde onde corpo, mente, e meio ambiente interagem entre si, fazendo-se necessário uma precisa avaliação das condições socioeconômicas, culturais, emocionais, familiar, dentre outras, para um correto diagnóstico das prováveis causas dos eventuais, aparecimento das doenças incidentes, o que rompe, portanto, com a visão uni-causal das doenças defendida no paradigma biomédico de saúde. Em outras palavras, o paradigma da promoção da saúde focaliza a atenção em aspectos mais amplos da comunidade que possam interferir nas condições de saúde das pessoas, sem se deixar levar única e exclusivamente pelas práticas médicas.

Entre as principais virtudes deste paradigma destacam-se:

- Favorece a interdisciplinaridade tratando os problemas de saúde em um perspectiva mais ampla, em que o indivíduo é abordado como um membro da comunidade em que está inserido, promovendo neste sentido, uma maior intervenção a favor da saúde.

- A saúde passa a ser vista como uma dimensão comunitária na qual sua manutenção não é obrigação exclusiva dos médicos, mais de toda comunidade desde um perspectiva coletiva e de cada pessoa de forma individualizada.

- Prioriza as ações preventivas, reduzindo os custos da atenção médica, ademais de evitar os riscos sociais que poderiam levar a morte ou a invalidez .

- É particularmente útil e aplicável a países em desenvolvimento como o Brasil, devido, muitas das doenças aqui existentes poderem ser superadas com medidas profiláticas simples e de baixo custo.

- Por ter um impacto imediato nas condições de vida das pessoas o paradigma da promoção da saúde melhora o bem-estar a qualidade de vida e o desenvolvimento social, pessoal e humano dos indivíduos de uma sociedade.

As debilidades mais relevantes deste modelo são:

- Consiste em um paradigma aparentemente muito 'estadista', no sentido de que o Estado seria o seu principal promotor, o que se contrapõe a tendência atual que enfatiza a redução do papel do Estado na promoção das políticas públicas sociais, entre elas as de saúde.

- Não enfatiza as pesquisas aplicadas que possam ajudar na busca de novos fármacos para antigas e novas doenças, neste caso, isso poderia desemborcar no desincentivo à pesquisa.

- Se fundamenta na base da hipótese de que as pessoas estão dispostas a participarem ativa e solidariamente na promoção de sua saúde, no entanto, na prática normalmente se tem

13 Ministério da saúde. Projeto Promoção da Saúde.. Brasília: Ministério da Saúde e Secretaria de políticas públicas, 2001. 
verificado que existem fortes resistências às mudanças, o que implica um desafio específico para os formuladores de políticas públicas de saúde.

- Normalmente existem compromissos por parte de alguns profissionais com um paradigma específico e isso pode ou não, favorecer a adoção de novas estratégias como a impulsionada pelo paradigma da promoção da saúde.

- A sua continuidade parece ainda ser incerta, devido sua grande dependência ao poder político local, que pode tornar esse tipo de estratégia fortemente influenciada por interesses políticos, eleitoreiros e clientelista.

- Depara-se com fortes barreiras e obstáculos, devido ainda predominar na nossa sociedade a cultura das ações curativas proposta pelo paradigma biomédico. Ademais, padece da escassez de médicos generalistas que se disponham a dedicarem suas atividades a esse tipo de programa.

\section{Conclusão}

Para os fins do presente estudo é importante constatar a existência de ao mesmo duas perspectivas epistemológicas ou paradigmas a partir dos quais é possível aproximar-se aos problemas de pesquisa de investigação e ao debate acadêmico especifico sobre a problemática tratada da saúde coletiva de maneira mais abrangente. Analisar estes e outros paradigmas desde a perspectiva da matriz disciplinaria é pertinente e perturbador, dado que cada uma das posições teóricos metodológicos ou paradigmas permite apreciar parcialmente a complexidade dos problemas de pesquisa próprios desta riquíssima temática.

Em tal sentido, a presente proposta de matriz disciplinaria para o estudo da fisioterapia coletiva está composta por paradigmas que oferecem explicações parciais, mais que definitivamente não conseguem esgotar os variados objetos de estudo das sub-especialidades. Nesta ordem de idéias, os paradigmas biomédico e da promoção da saúde, assim como outras posições teórico-metodológicas que não foram consideradas neste estudo, mais que poderão ser integradas na matriz no futuro, gozam de uma considerável capacidade explicativa, mais também de limitações operacionais. Em conseqüência, desde o ponto de vista teórico, os pesquisadores na área da ciências da saúde, na disciplina da saúde coletiva, podem e devem enfrentar com entusiasmo o duplo desafio de dominar o debate epistemológico que se apresenta na matriz disciplinaria especifica e, logo apresenta-se os problemas objetos de investigação propriamente dito.

Finalmente, uma vez considerada a capacidade explicativa dos paradigmas que formam parte da matriz disciplinaria especifica da saúde coletiva, tem-se optado por assumir o paradigma da promoção da saúde como instrumento de mudanças e transformação dentro do setor saúde. 